\title{
Dunning rat prostate adenocarcinomas and alternative splicing reporters: powerful tools to study epithelial plasticity in prostate tumors in vivo
}

\author{
Sebastian Oltean · Phillip G. Febbo • \\ Mariano A. Garcia-Blanco
}

Received: 10 March 2008/ Accepted: 21 May 2008/Published online: 4 June 2008

(C) The Author(s) 2008

\begin{abstract}
Using alternative splicing reporters we have previously observed mesenchymal epithelial transitions in Dunning AT3 rat prostate tumors. We demonstrate here that the Dunning DT and AT3 cells, which express epithelial and mesenchymal markers, respectively, represent an excellent model to study epithelial transitions since these cells recapitulate gene expression profiles observed during human prostate cancer progression. In this manuscript we also present the development of two new tools to study the epithelial transitions by imaging alternative splicing decisions: a bichromatic fluorescence reporter to evaluate epithelial transitions in culture and in vivo, and a luciferase reporter to visualize the distribution of mesenchymal epithelial transitions in vivo.
\end{abstract}

Keywords DT · AT3 - Dunning · FGFR2 .

Alternative splicing $\cdot$ EMT $\cdot$ MET $\cdot$ Imaging

Electronic supplementary material The online version of this article (doi:10.1007/s10585-008-9186-y) contains supplementary material, which is available to authorized users.

S. Oltean · P. G. Febbo · M. A. Garcia-Blanco $(\bowtie)$

Department of Molecular Genetics and Microbiology, Duke University Medical Center, Research Drive, Box 3053

(424 CARL), Durham, NC 27710, USA

e-mail: garci001@mc.duke.edu

S. Oltean - M. A. Garcia-Blanco

Center for RNA Biology, Duke University Medical Center,

Durham, NC 27710, USA

P. G. Febbo

Institute of Genome Science and Policy, Duke University

Medical Center, Durham, NC 27710, USA

P. G. Febbo · M. A. Garcia-Blanco

Department of Medicine, Duke University Medical Center,

Durham, NC 27710, USA

\section{Introduction}

Examination of the human genome reveals a preponderance of complex genes that produce equally intricate primary transcripts [1-3]. In humans, a typical pre-messenger RNA (pre-mRNA) contains seven or eight introns, each averaging more than 3,000 nucleotides in length, and eight or nine exons, which average only 300 nucleotides, with the average internal exon measuring only 145 nucleotides [1, 2]. Components of the spliceosome (U snRNPs and general splicing factors) recognize intronic and exonic sequence elements that critically define exon-intron boundaries and mediate the removal of introns and ligation of exons in the process known as pre-mRNA splicing. Alternative RNA splicing is the process by which a primary transcript yields different mature RNAs leading to the production of protein isoforms with diverse and even antagonistic functions [4-9]. The annotation of mammalian genomes reveals that the bulk of intron-containing transcripts are alternatively spliced [10-13]. Many different forms of alternative splicing together with differential use of promoters and $3^{\prime}$ cleavage/polyA sites leads to staggering versatility among transcripts driven by RNAP II. Most importantly, alternative splicing is one of the several mechanisms by which broad programs of gene expression are established in different cell types.

An elegant example of regulated alternative splicing is the choice between IIIb and IIIc isoforms of FGFR2. Alternative inclusion of exon 8 (IIIb) or exon 9 (IIIc) leads to the expression of FGFR2(IIIb) and FGFR2(IIIc), respectively. This decision is cell-type specific, most epithelial cells express the FGFR2(IIIb) isoform exclusively, and most mesenchymal cells express solely FGFR2(IIIc). The alternative use of exons IIIb or IIIc alters ligand binding [14, 15] and its regulation is required for normal development in mice 
and humans [16-18]. The status of FGFR2 alternative splicing depends on the interplay between several cis-acting elements in the FGFR2 pre-mRNA and trans-acting factors, some of which are cell-type specific. Two cell-type specific cis-acting elements, intronic activating sequence 2 (IAS2) and intronic splicing activator and repressor (ISAR, also known as IAS3), which form a stem, collaborate to activate exon IIIb and repress exon IIIc in an epithelial-specific manner [19-23]. Additionally, we identified two gcaug sequence elements, located downstream of ISAR and within exon IIIc, and showed that these play a role in epithelial exon IIIc repression [22]. Moreover, we showed that splicing factors of the Fox family of proteins are responsible for this repression of exon IIIc in epithelial cells [24]. The Fox family of proteins, and in particular Fox-2, is a likely candidate for a master regulator of FGFR2 splicing during epithelial plasticity.

The differential splicing of FGFR2 transcripts underscores large differences in gene expression programs in epithelial and mesenchymal cells. Epithelial cells are tightly connected to each other by specialized structures on their surface and form well organized apical-basal polarized layers [25]. Mesenchymal cells, on the other hand, do not form well-organized layers, are motile, and have a front-back polarity. Epithelial cells can become mesenchymal cells and vice versa via phenotypic transitions, known as epithelial-mesenchymal (EMT) and mesenchymal-epithelial transitions (MET). These are critical for vertebrate ontogeny and have been proposed to play important roles in tumorigenesis [25-28]. Indeed, the evidence that these epithelial transitions are seen in prostate cancer is now overwhelming, and the data that support their importance are compelling [29-32].

In this manuscript we build upon our previous observation that cells in Dunning AT3 rat prostate tumors show heterogeneity in the alternative splicing of FGFR2 transcripts and this is due to MET [29]. We demonstrate here that the Dunning DT and AT3 cells, which express epithelial and mesenchymal markers, respectively, represent an excellent model to study epithelial transitions in prostate cancer. Moreover, we introduce two new tools to study the epithelial transitions by imaging alternative splicing decisions: a bichromatic fluorescence reporter to evaluate epithelial transitions in culture and in vivo, and a luciferase reporter to visualize the distribution of MET in vivo.

\section{Materials and methods}

Gene expression analysis

Triplicate cultures of AT3 and DT cells were grown to $60 \%$ confluency. Total RNA was isolated using the RNeasy kit (Qiagen, Inc.) and triplicate samples were submitted to the Duke Microarray Facility. Gene expression analysis was performed using the RO27 K rat spotted arrays 3.0 (Operon, Inc.). Bioinformatical analysis of expression differences between AT3 and DT cells was done using the GeneSpring GX software version 7.3.1 (Agilent Technologies, Inc.). The data files (representing signals for 26,986 gene probes in all six data points, three for AT3 and three for DT) were normalized using the feature: per Spot and per Chip-intensity dependent (lowess) normalization. The resulting gene list was used to determine the significantly differentially expressed genes between AT3 and DT using the "Filtering on Volcano plot" feature with the following characteristics: (1) Test type: parametric test, don't assume variances equal; (2) Multiple testing correction: none; (3) Fold Difference: 2fold or greater and a $P$-value cutoff of 0.01 .

Gene set enrichment analysis

To infer differential pathway activity from gene expression differences between the AT3 and DT cells, gene set analysis was performed (GSEA) [33]. Briefly, we first generated a gene set from microarray features from the R027 K rat spotted array (v 3.0) with increased expression in the AT-3 cells compared to the DT cells using an a priori established cut off of $\geq 2$-fold increase in average expression. The features identified using the rat array were mapped to Affymetrix U133A accession numbers using annotation files provided by Operon (See Supplemental Table 1). Differential enrichment of this gene set was then tested across a cohort of local $(n=16)$ and metastatic $(n=$ 32) prostate cancer tumors using previously published data [34]. The statistical significance of differential enrichment in one class (metastatic) vs. the other (local) was determined using a weighted Kolmogorov-Smirnoff metric to determine an enrichment score (ES), and random permutation of phenotypic labels was performed to compare the observed results with reiterative random permutations of class labels to establish a false discovery rate (FDR). Parameters for GSEA included: (1) genes were ranked according differential expression between metastatic (up) and local (down) prostate samples using a Student's t-test, (2) weighted analysis was performed with the weight assigned a value of 1.0, and (3) 1,000 random permutations of randomized class labels were used to establish the FDR.

\section{Bichromatic fluorescent reporter pRGIIIc}

The minigene pRGIIIc was constructed on the backbone of minigene RG6 [35] kindly provided by Dr Thomas Cooper (Baylor College of Medicine). The exon IIIc and flanking intronic regions of the FGFR2 gene were amplified from the pRIIIcI $^{2}$ minigene [29] and inserted between the XbaI and 
AgeI sites of the RG6 minigene. A 21 nucleotide region encoding a nuclear localization signal in the first exon of the RG6 minigene was deleted. Complete sequence of the plasmid will be provided upon request. AT3 and DT rat prostate tumor cells were kindly provided to our laboratory by Dr. Wallace McKeehan (Texas A\&M University Health Sciences Center, Houston, TX). Cells were transfected using lipofectamine (Invitrogen Corporation, Inc.) and stable transfectants were selected using $500 \mu \mathrm{g} / \mathrm{ml} \mathrm{G} 418$.

\section{Luciferase reporters}

The minigenes pFFint and $\mathrm{pFFIIIcI}^{2}$ are identical to $\mathrm{pRint}$ and pRIIIcI $^{2}$ (described elsewhere [29]) except for having the firefly luciferase ORF instead of the RFP ORF. Complete sequence of the plasmids will be provided upon request. AT3 cells were transfected using lipofectamine (Invitrogen Corporation) and stable transfectants were selected using $15 \mu \mathrm{g} / \mathrm{ml}$ blasticidin.

Animals and tumor cells implantation

AT3 cells were trypsinized, resuspended in PBS to a final concentration of $10^{6} / \mathrm{ml}$ and kept on ice. $3 \times 10^{5}$ cells were injected subcutis (sc) in the flanks of Copenhagen 2331 rats (Harlan, Inc.) (75-90 g and $\sim 2$ months in age). Animals were continuously monitored for tumor growth. All animal procedures were performed according to the Duke University Institutional and Animal Care and Use guidelines.

\section{Bioluminescence imaging}

For cell culture studies D-luciferin was added to the culture medium (final concentration $50 \mu \mathrm{g} / \mathrm{ml}$ ) and 10 min later imaging was performed using the IVIS imaging system (Xenogen). For animal in vivo imaging, following anaesthesia, rats were injected intraperitonealy with D-luciferin $(50 \mathrm{mg} / \mathrm{kg})$ and $10 \mathrm{~min}$ later sequential imaging was performed as described.

\section{Results and discussion}

The Dunning DT and AT3 cell lines as a model to study epithelial transitions in prostate cancer

We have previously studied the alternative splicing of FGFR2 transcripts in DT (aka DT3) and AT3 cells, which are two cell lines derived from the well-differentiated and androgen sensitive Dunning R-3327 rat prostate tumor [3640]. The indolent DT tumor cells express FGFR2(IIIb) like normal prostate epithelium, while AT3 cells have switched to the FGFR2(IIIc) isoform [38]. The significance of these alternative decisions for tumor behavior is underscored by the fact that forced expression of FGFR2(IIIb) suppresses tumor progression of AT3 tumors [41]. More recently, we have developed fluorescence reporters that permit us to image FGFR2 alternative splicing decisions in culture and in vivo (in normal tissues and tumors) [29, 42, 43]. Our data suggested that the DT and AT3 cells could be a useful system to evaluate epithelial transitions in the context of prostate cancer, with the latter being the result of a prior EMT. Indeed, DT cells expressed E-cadherin, a marker of epithelial cells, and this protein was seen to be in association with the plasma membrane, while in AT3 cells Ecadherin was undetectable by either Western blots or immunofluorescence (Fig. 1 and data not shown). This result did not depend on the confluency of cells (data not shown). When we stained for Vimentin, which is a mesenchymal marker, DT cells were uniformly negative, but AT3 cells showed heterogeneous staining, with about $10 \%$ of the cells showing bright staining (data not shown). A previous study that included AT3 and DT cell lines and other lines from the Dunning prostate carcinoma system found a progressive decrease in keratin and increase in Vimentin staining from well-differentiated and low-metastatic (like DT) to anaplastic and highly metastatic lines (like AT3) [44]. These data and ours are consistent with the DT and AT3 cells representing two points in the epithelialmesenchymal axis.

To further test this idea we carried out en masse gene expression analysis in DT and AT3 cells. Total RNA was extracted from three independent cultures of DT and AT3 each and transcript levels were quantified using the RO27 K rat spotted arrays 3.0 (Operon, Inc.) (https://www.operon. com/arrays/OpArray_rat.php). The complete list of gene products that were overexpresed by $>2$-fold $(P<0.01)$ is presented in Supplemental Table 2. DT cells were shown to express $\sim 1,000$-fold greater levels of Cingulin, Integrin- 36 and Zyxin transcripts than did AT3 cells (Table 1). These gene products have been associated with interactions between epithelial cells [45]. Several other epithelial plasticity related transcripts, including the RNA for E-cadherin, were also over-represented by $\sim 16$ to $\sim 300$-fold in DT cells relative to AT3 cells (Table 1). The expression analysis was consistent with DT as defining an epithelial-like phenotype in the plasticity axis. There were, however, some overexpressed gene products that are not expected in normal epithelial cells (e.g., Mmp9) (italicized in Table 1). Whereas we cannot fully explain the increased expression of these gene products in DT relative to AT3 cells, it is important to note that DT cells are not normal epithelial cells but rather transformed cells [36]. Moreover, MMP9 was also reported to have increased expression in an "epithelial" cell line derived from transitional cell carcinoma of the bladder [46]. 
Fig. 1 DT cells show high levels of expression and predominantly junctional distribution of E-cadherin compared to AT3 cells. Overlay of E-cadherin

immunofluorescence and Hoechst staining of the same fields (left panels) as well as phase contrast images (right panels) for both DT and AT3 cells. E-Cadherin antibody is labeled with AlexaFluor 555 and images were acquired at $200 \times$ magnification

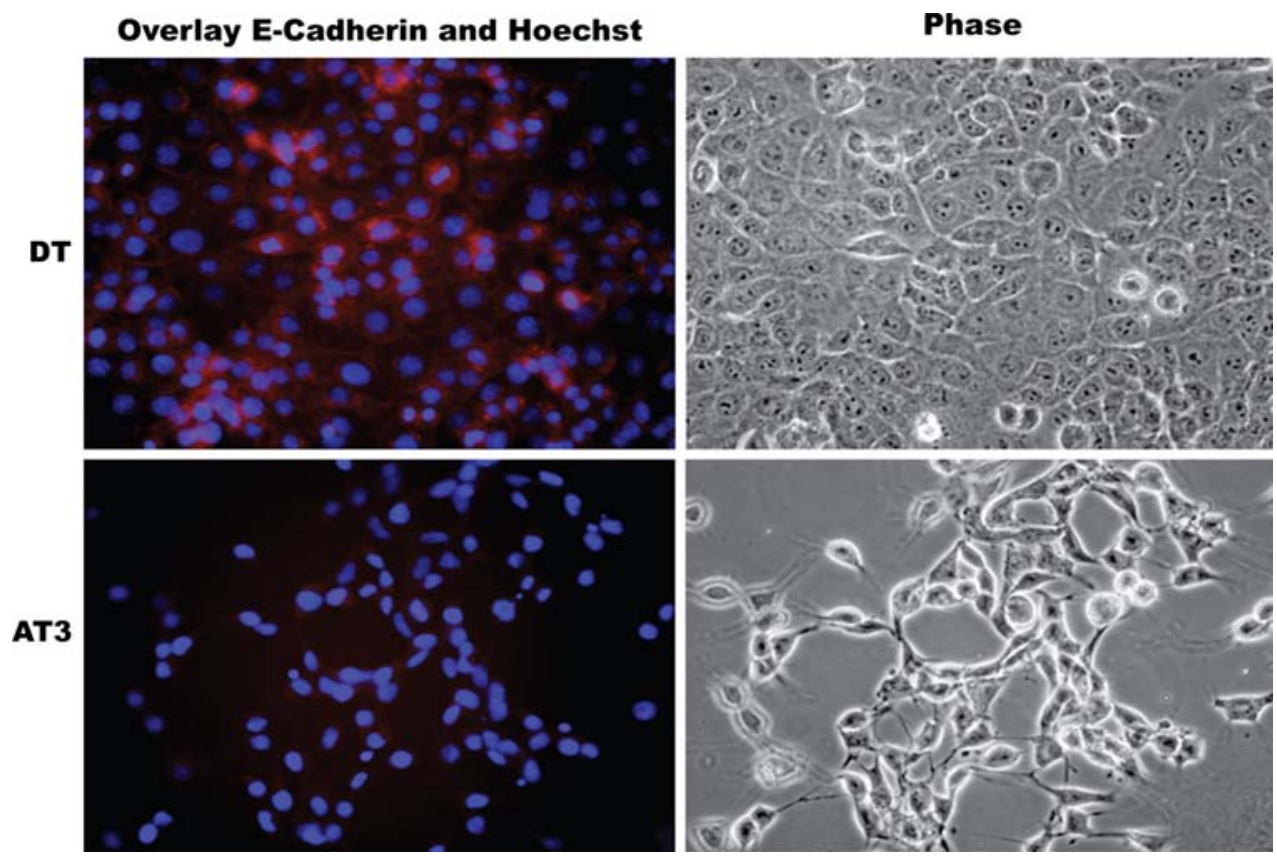

Table 1 Epithelial plasticity related transcripts overexpressed in DT relative to AT3 cells

\begin{tabular}{llll}
\hline Fold AT3/DT & Common gene name & Genbank (Rat) & Description \\
\hline 0.060 & CLI; APOJ; SGP2; SGP-2; & SP-40; Trpm2; Trpmb; TRPM-2; & M16975 \\
& TRPM2B; RATTRPM2B & & \\
& Igfbp7 & BC086582 & Insulin-like growth factor binding protein 7 \\
0.017 & Notch3 & AF164486 & Notch gene homolog 3 \\
0.012 & Cldn4 & BC083582 & Claudin 4 \\
0.008 & Cx26; CXN-26; MGC93804 & BC078952 & Gap junction membrane channel protein beta 2 \\
0.003 & Lisch7; MGC124592 & AF119667 & Lipolysis stimulated lipoprotein receptor \\
0.003 & C4.4a & AJ001043 & Ly6/Plaur domain containing 3 \\
0.003 & LOC304000 & BC086342 & Cell adhesion molecule JCAM \\
0.003 & Cdh1 & AB017696 & Cadherin 1 (E-cadherin) \\
0.003 & MGC93307 & BC081757 & Growth factor receptor bound protein 7 \\
0.003 & Mmp9 & U24441 & Matrix metallopeptidase 9 \\
0.002 & Zyx & BF401563 & Zyxin \\
0.001 & MGC94023 & BC079069 & Integrin, beta 6 \\
0.001 & Cgn_predicted & CK596641 & Cingulin (predicted) \\
0.001 & & &
\end{tabular}

Examination of transcripts that were represented in AT3 showed a dramatic signature consistent with EMT: the regulators Twist and Snail RNAs were both upregulated $\sim 280$ and $\sim 15$-fold, respectively, and Vimentin was upregulated $\sim 24$-fold (Table 2). This was consistent with results from detection of Vimentin protein in AT3 cells in culture above and also in AT3 tumors in vivo [29]. As noted for the DT cells there was a transcript associated with epithelial plasticity, the RNA for Syndecan 2, which is not expected in EMT (see Discussion below). It should be emphasized here that epithelial-mesenchymal differences cannot account for all of the differences in gene expression between DT and AT3 cells. Nonetheless, the overall gene expression pattern of transcripts associated with epithelial plasticity suggested that the transition of the Dunning tumors from DT to AT3 cells involved an EMT.

In order to evaluate the relevance of Dunning model to human prostate cancer, we compared the gene expression profiles in these cells with that in human prostate tumors and metastases. In order to do this, we used Gene Set Enrichment Analysis (GSEA) [33] and compared our data to that from patient specimens [34]. GSEA is a powerful 
Table 2 Epithelial plasticity related transcripts overexpressed in AT3 relative to DT cells

\begin{tabular}{clll}
\hline Fold AT3/DT & Common gene name & Genbank (Rat) & Description \\
\hline 1583.0 & Bmp2 & Z25868 & Bone morphogenetic protein 2 \\
522.1 & Igfbp4 & CO403206 & Insulin-like growth factor binding protein 4 \\
279.5 & Twist & AF266260 & Twist gene homolog 1 \\
138.2 & Sema3a & X95286 & Sema domain, immunoglobulin domain (Ig), \\
& & & short basic domain, secreted, (semaphorin) 3A \\
121.0 & HSPG & BC070890 & Syndecan 2 \\
23.8 & Vim & BC061847 & Vimentin \\
14.9 & Sna & BC079210 & Snail homolog 1 \\
\hline
\end{tabular}

way of matching a defined gene set to the rank order of another en masse gene expression experiment. The effectiveness of GSEA hinges on the ability to quantify and visualize the distribution of the defined gene set within the data of another microarray evaluation. By relying on the distribution, GSEA is not affected by varying fold change between cell types. The software determines if genes in a set (in this case the genes with $>2$-fold average expression in AT3 when compared to DT) occur more frequently at the top or bottom of a list of genes ranked according to their differential expression across the phenotype of interest (metastatic vs. local prostate cancer). The program provides an enrichment score (ES) based on a weighted Kolmogorov-Smirnov statistic and provides a false discovery rate (FDR) based upon the value of the observed ES compared to the distribution of ES across 1,000 randomly permutations of phenotypic labels [33].

We found significant enrichment for the human metastatic pattern among genes that were overexpressed $\geq 2$ fold in AT3 when compared to DT cells (Enrichment Score was 0.25 , normalized ES of 1.24) and the false discovery rate was 0.12 (recommended significant range $<0.25$ [33]) (Fig. 2). These data strongly suggest that basic features of tumor behavior studied in the Dunning prostate adenocarcinomas will be observed in human prostate cancers. These data could also be taken to support the idea that the more mesenchymal cell line (AT3) was closer in gene expression pattern to metastases in humans (see Discussion below).

A bichromatic reporter can image splicing differences in epithelial and mesenchymal cells

We modifed the elegant RG6 bichromatic reporter designed by Orengo et al. [35] to obtain pRGIIIc, a construct to report on the inclusion or skipping of the rat FGFR2 exon IIIc (Fig. 3a). The plasmid pRGIIIc incorporated elements from previous reporters created in the laboratory [21, 22, 24, 47, 48]. RGIIIc transcripts, like FGFR2 transcripts, preferentially include exon IIIc in AT3 cells (mesenchymal-like) (Fig. 3b) and this transcript codes for EGFP (see schematic

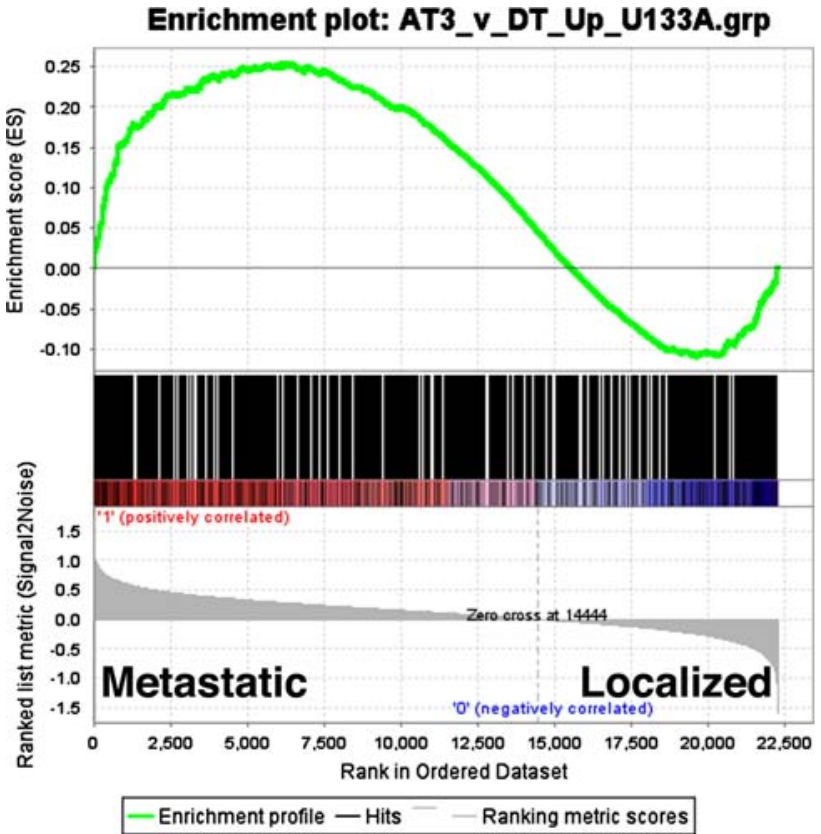

Fig. 2 The Dunning tumor cell lines DT and AT3 recapitulate differences in gene expression observed during tumor progression in human prostate cancer. GSEA output showing the distribution of the gene set with increased expression in the AT3 cells compared to the DT cells using an a priori established cut off of $\geq 2$-fold increase in average expression. Differential enrichment of this gene set was then tested across a cohort of localized $(n=16)$ and metastatic $(n=32)$ human prostate cancer tumors using previously published data [34]

in Fig. 3a). Indeed, AT3 cells harboring pRGIIIc express EGFP (Fig. 3c, d). It should be noted that RT-PCR analysis detected low levels of RGIIIc transcripts that skip exon IIIc or that use a cryptic site within exon IIIc, which produces what we called a mini-IIIc exon [48]. Nonetheless, these levels of transcripts did not result in detectable levels of RFP (Fig. 3c, d). When we modified the reporter, we deleted a nuclear localization signal inserted by Orengo et al, however, the protein that includes sequences coded by exon IIIc still localizes to the nucleus of AT3 cells (Fig. 3c). This serendipitous result was useful when analyzing the dual expression of the reporter as will be discussed below. 

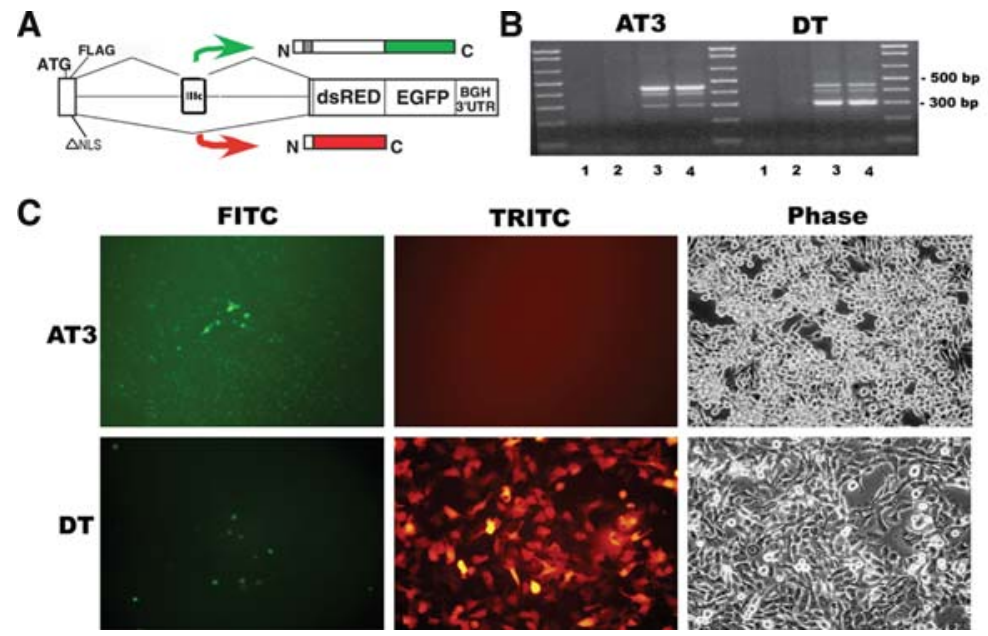

D
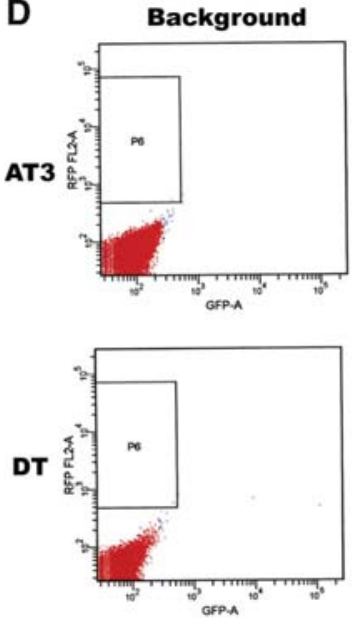

Stable lines
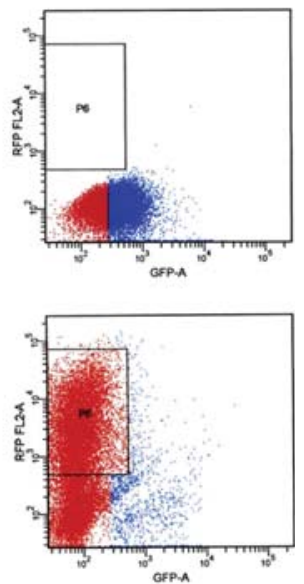

Fig. 3 Bichromatic fluorescence RGIIIc construct to report on EMT and MET. (a) Schematic of the dual reporter (adapted from Orengo et al. [35]). Inclusion of exon IIIc of FGFR2 results in a fusion protein in frame with EGFP while skipping of this exon results in RFP expression. (b) RT-PCR analysis of total RNA from AT3 and DT cells stably transfected with RGIIIc resulted in the expected size products (446 bp for products that include exon IIIc and $301 \mathrm{bp}$ for products that skip this exon). The middle-size band was sequenced and represents a product that includes "mini IIIc", which lacks the

RGIIIc transcripts, like endogenous FGFR2 transcripts, preferentially skipped exon IIIc in DT cells (epithelial-like) (Fig. 3b) and this $\triangle \mathrm{IIII}$ transcript codes for RFP (see schematic in Fig. 3a). Indeed DT cells harboring pRGIIIc expressed RFP, which distributes to both the nucleus and the cytoplasm of these cells, (Fig. 3c, d). Again the analysis of RNA species suggested that there was low level of inclusion of IIIc and mini-IIIc exons. It is possible that few of the DT cells include exon IIIc more efficiently and result EGFP expression (Fig. 3c, d); this result could be taken to suggest epithelial plasticity among DT cells in culture. Indeed overlay images indicated that the DT cells expressing EGFP did not express RFP (data not shown) (see Discussion). The data shown above demonstrate the utility of pRGIIIc alternative splicing reporter as a sensor of epithelial plasticity.

\section{Alternatively spliced bioluminescence reporters can image mesenchymal-epithelial transitions in lung metastases}

The work above and work previously published [29] highlighted the utility of fluorescence-based alternative splicing reporters to detect epithelial-mesenchymal transitions. These reporters have permitted us to observe these transitions ex vivo (tumors and lungs were dissected, sectioned and imaged by epifluorescence microscopy). In order to expand our work to detect these transitions in animals in vivo we designed bioluminescence alternative last 50 bases of the IIIc exon due to an alternative $5^{\prime}$ splice site we recently described (see [48]). Lanes are: 1, RT from naïve cells; 2, PCR without RT from AT3-RGIIIc or DT-RGIIIc cells; 3 and 4, duplicates of RT-PCR reactions from AT3-RGIIIc or DT-RGIIIc cells. (c) Fluorescence and phase-contrast imaging of AT3-RGIIIc and DT-RGIIIc cells (images were acquired at $200 \times$ magnification). (d) Flow cytometric analysis of AT3 and DT cells (left panels) and AT3-RGIIIc and DT-RGIIIc cells (right panels)

splicing reporters. The design was similar to the fluorescence reporters with which we have previously observed the tumor cells plasticity [29]. The minigene pFFint, which is used as a control, contains a constitutive intron and was predicted to express firefly luciferase in both DT and AT3 cells (Fig. 4a). On the other hand $\mathrm{pFFIIIcI}^{2}$ should produce luminescence signal only in cells that skip the FGFR2 exon IIIc (Fig. 4a). Indeed, AT3 cells expressed firefly luciferase efficiently from FFint transcripts but did not from FFIIICI ${ }^{2}$ RNAs (Fig. 4a). On the other hand DT cells expressed luciferase efficiently from FFIIIcI ${ }^{2}$ RNAs (Fig. 4b). These data suggested that these novel reporters could be used to visualize epithelial status in vivo.

To test this potential, we decided to examine the behavior of AT3 tumors bearing these bioluminescence reporters. Whereas AT3 cells in culture and the great majority of these cells in tumors display a mesenchymal phenotype, we previously noted that clusters of cells in tumors and lung metastasis undergo mesenchymalepithelial transitions (MET) [29]. We injected one Copenhagen 2331 rat $s c$ in both flanks with AT3 cells stably transfected with pFFint and three animals were injected $s c$ in both flanks with AT3 cells stably transfected with $\mathrm{pFFIIIcI}^{2}$. Tumors were left to grow and appearance of metastasis in lungs was monitored by in vivo luminescence imaging every other day. In all four animals, luminescence-reporters AT3 cells were mixed with AT3 cells stably expressing EGFP, so metastasis observed with luminescence could be confirmed at microscopic level. 
A

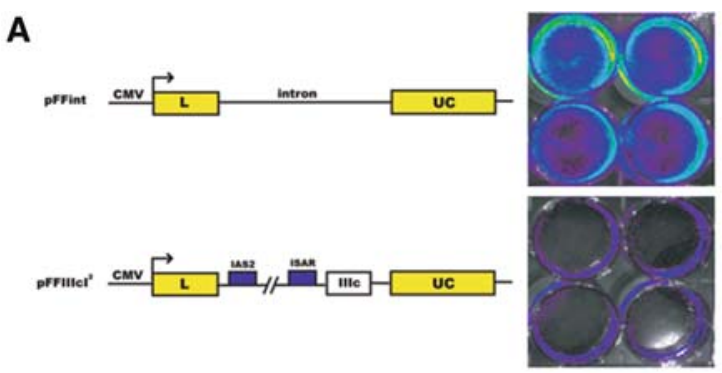

B

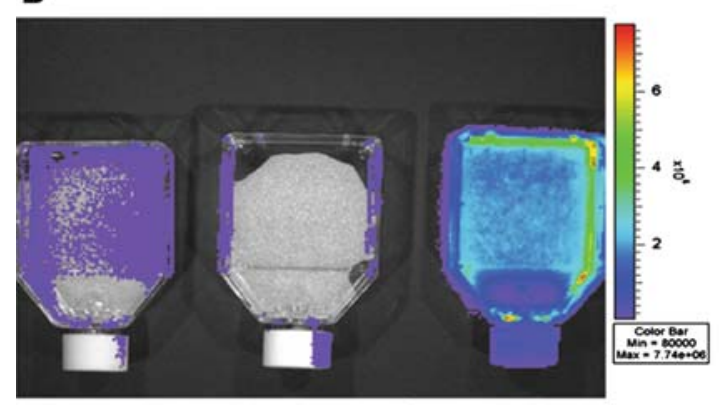

C

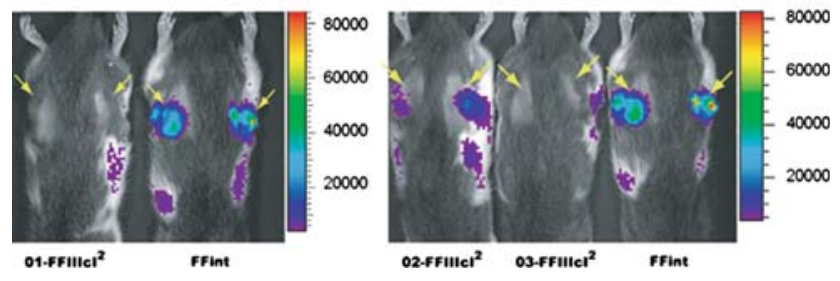

Fig. 4 Bioluminescence reporters and in vivo bioluminescence imaging of MET. (a) (Left) Schematic of bioluminescence minigene reporter constructs $\mathrm{pFFIIIcI}^{2}$ and $\mathrm{pFFint}$ (see Materials and methods). (Right) Bioluminescence imaging of AT3 cells stably transfected with the pFFIIIcI $^{2}$ and pFFint. (b) Bioluminescence imaging of AT3 cells stably transfected with pFFint (left), AT3 cells stably transfected with pFFIIIcI $^{2}$ (middle) and DT cells stably transfected with pFFIIIcI $^{2}$ (right). Each flask was seeded with 1 million cells the day before images were acquired. (c) Bioluminescence images of three different animals having FFIIICI ${ }^{2}$ tumors in both flanks (left animal in the left panel; left and middle animals in the right panel) and the same animal with control FFint tumors in both flanks (right animal in both panels) taken 13 days after subcutaneous injection of tumor cells. The yellow arrows indicate positions of the flank tumors. The color scales represent the signal intensities at the surface of the animals in photons $/\left(\mathrm{cm}^{2} \mathrm{~s}\right.$ steradian). Minimum and maximum thresholds (after background substraction) are also indicated. In all cases photons were counted for $120 \mathrm{~s}$. The image on the right was taken $20 \mathrm{~min}$ after injection of luciferin while the image on the left was taken $40 \mathrm{~min}$ after injection

Thirteen days following implantation, strong luminescence signal was seen in the two primary tumors of the FFint animal and much weaker luminescence was detected in the lungs (Fig. 4c; two images of the same animal (FFint) are shown in the two panels). Only three of the six FFIIIcI $^{2}$ tumors had detectable luminescence (on both flanks of animal 02-FFIIIcI ${ }^{2}$ and on the left flank of animal $03-$ FFIIICI $^{2}$ ). This is consistent with our previous ex vivo observations using fluorescence reporters [29] and we interpret the (low level) luminescence in the FFIIIcI ${ }^{2}$ primary tumors as originating in MET foci (Fig. 4c). Interestingly, the intensity of the signal from the reporters observed in the lung metastases relative to the signal from the primary tumors was higher for FFIIIcI $^{2}$ tumors (Fig. 4c). Indeed in the case of the one animal (01-FFII$\mathrm{IcI}^{2}$ - Fig. 4c), only cells metastatic to the lung expressed luciferase, and thus had undergone MET. These observations suggested that MET was more frequent in metastases as we had predicted based on our ex vivo analysis [29]. Moreover, these data show that the luminescence reporters can be used to observe alternative splicing changes in vivo during tumor progression and metastasis.

EMT and MET transitions have been described before and are thought to play an important role during development, when cells activate or repress different gene expression programs in order to migrate at a certain point in time and space and later become stationary [25, 49, 50]. There is an increasing number of reports showing that cells at the edge of carcinomas or metastatic cells are able to reactivate EMT and MET programs in order to successfully complete the metastasis program [28, 49]. Nonetheless, skepticism still exists in the field about the existence of these transitions during tumor invasion and metastasis, especially because of the lack of evidence in vivo [51]. In previous work, we have shown that the AT3 cells, which have been previously described as mesenchymal-like [38, 52], display surprising plasticity during tumor growth and metastasis [29]. Using alternative splicing reporters we observed MET occurring both in tumors (especially near stroma) and in lung metastasis around blood vessels. The previous observations were done ex vivo, by excising the tumors and metastatic lungs and analyzing sections under epifluorescence microscopy. In this work, to our knowledge, we are the first to show that MET associated with tumor growth and metastasis can be visualized in a living animal. Using alternatively spliced luciferase reporters that have a very low signal when cells display a mesenchymallike phenotype and high signal when cells turn to a more epithelial-phenotype we show that it is possible to follow MET in vivo in living animals. Although we have not ruled out that a very small population of cells could have transitioned in the culture dish, and expanded only after implantation, we believe that the data shown here and our previously published data are much more likely due to epithelial transitions occurring post-implantation. Regardless, we have developed tools that can be used to study the contribution of MET to metastasis in vivo in this particular system. Additionally, here we show the first generation of bichromatic reporters that can sense both EMT and MET.

We propose that Dunning tumor cells (both DT and AT3) might be in metastable phenotypic states. Indeed the expression of Mmp9 and other mesenchymal gene products 
at higher levels in DT cells than AT3 cells and vice versa for Syndecan 2 in AT3 cells may reflect this metastability. Indeed the expression of EGFP by a small number of DT cells transfected with the pRGIIIc bichromatic reporter is consistent with this idea. We posit that the ability of these cells to toggle between phenotypic states contributes to malignancy. Whereas we make our proposal based on a rat model system for prostate cancer, our comparison of the Dunning cells with human specimens strongly suggests that our hypothesis has relevance to human cancers.

Acknowledgements M. A. G-B acknowledges support from NIH (NCI and NIGMS) grants R33 CA97502 and RO1 GM063090. P.G.F. is a Damon Runyon-Lily Clinical Investigator (CI-29-05) and acknowledges support from the DOD (PC060879). We thank Dr. Robert Brazas and Mr. Todd Albrecht for construction of FFint and FFIIICI $^{2}$.

Open Access This article is distributed under the terms of the Creative Commons Attribution Noncommercial License which permits any noncommercial use, distribution, and reproduction in any medium, provided the original author(s) and source are credited.

\section{References}

1. Venter JC, Adams MD, Myers EW et al (2001) The sequence of the human genome. Science 291(5507):1304-1351. doi:10.1126/ science. 1058040

2. Lander ES, Linton LM, Birren B et al (2001) Initial sequencing and analysis of the human genome. Nature 409(6822):860-921. doi: $10.1038 / 35057062$

3. ENCODE Project Consortium et al (2007) Identification and analysis of functional elements in $1 \%$ of the human genome by the ENCODE pilot project. Nature 447(7146):799-816. doi: 10.1038 /nature 05874

4. Black DL (2003) Mechanisms of alternative pre-messenger RNA splicing. Annu Rev Biochem 72:291-336. doi:10.1146/annurev. biochem.72.121801.161720

5. Garcia-Blanco MA, Baraniak AP, Lasda EL (2004) Alternative splicing in disease and therapy. Nat Biotechnol 22(5):535-546. doi:10.1038/nbt964

6. Stetefeld J, Ruegg MA (2005) Structural and functional diversity generated by alternative mRNA splicing. Trends Biochem Sci 30(9):515-521. doi:10.1016/j.tibs.2005.07.001

7. Stamm S, Ben-Ari S, Rafalska I et al (2005) Function of alternative splicing. Gene 344:1-20. doi:10.1016/j.gene.2004.10.022

8. Matlin AJ, Clark F, Smith CW (2005) Understanding alternative splicing: towards a cellular code. Nat Rev Mol Cell Biol 6(5):386-398. doi:10.1038/nrm1645

9. Zavolan M, van Nimwegen E (2006) The types and prevalence of alternative splice forms. Curr Opin Struct Biol 16(3):362-367. doi:10.1016/j.sbi.2006.05.002

10. Modrek B, Lee CJ (2003) Alternative splicing in the human, mouse and rat genomes is associated with an increased frequency of exon creation and/or loss. Nat Genet 34(2):177-180. doi: $10.1038 / \operatorname{lng} 1159$

11. Johnson JM, Castle J, Garrett-Engele P et al (2003) Genome-wide survey of human alternative pre-mRNA splicing with exon junction microarrays. Science 302(5653):2141-2144. doi: 10.1126/science. 1090100
12. Resch A, Xing Y, Alekseyenko A et al (2004) Evidence for a subpopulation of conserved alternative splicing events under selection pressure for protein reading frame preservation. Nucleic Acids Res 32(4):1261-1269. doi:10.1093/nar/gkh284

13. Blencowe BJ (2006) Alternative splicing: new insights from global analyses. Cell 126(1):37-47. doi:10.1016/j.cell.2006. 06.023

14. Plotnikov AN, Hubbard SR, Schlessinger J et al (2000) Crystal structures of two FGF-FGFR complexes reveal the determinants of ligand-receptor specificity. Cell 101(4):413-424. doi:10.1016/ S0092-8674(00)80851-X

15. Yeh BK, Igarashi M, Eliseenkova AV et al (2003) Structural basis by which alternative splicing confers specificity in fibroblast growth factor receptors. Proc Natl Acad Sci USA 100(5):22662271. doi:10.1073/pnas.0436500100

16. Hajihosseini MK, Wilson S, De Moerlooze L et al (2001) A splicing switch and gain-of-function mutation in FgfR2-IIIc hemizygotes causes Apert/Pfeiffer-syndrome-like phenotypes. Proc Natl Acad Sci USA 98(7):3855-3860. doi:10.1073/pnas. 071586898

17. De Moerlooze L, Spencer-Dene B, Revest J et al (2000) An important role for the IIIb isoform of fibroblast growth factor receptor 2 (FGFR2) in mesenchymal-epithelial signalling during mouse organogenesis. Development 127(3):483-492

18. Wilkie AO, Patey SJ, Kan SH et al (2002) FGFs, their receptors, and human limb malformations: clinical and molecular correlations. Am J Med Genet 112(3):266-278. doi:10.1002/ajmg.10775

19. Del Gatto F, Plet A, Gesnel MC et al (1997) Multiple interdependent sequence elements control splicing of a fibroblast growth factor receptor 2 alternative exon. Mol Cell Biol 17(9): 5106-5116

20. Carstens RP, McKeehan WL, Garcia-Blanco MA (1998) An intronic sequence element mediates both activation and repression of rat fibroblast growth factor receptor 2 pre-mRNA splicing. Mol Cell Biol 18(4):2205-2217

21. Wagner EJ, Curtis ML, Robson ND et al (2003) Quantification of alternatively spliced FGFR2 RNAs using the RNA invasive cleavage assay. RNA 9(12):1552-1561. doi:10.1261/rna.5840803

22. Baraniak AP, Lasda EL, Wagner EJ et al (2003) A stem structure in fibroblast growth factor receptor 2 transcripts mediates celltype-specific splicing by approximating intronic control elements. Mol Cell Biol 23(24):9327-9337. doi:10.1128/MCB.23.24. 9327-9337.2003

23. Mistry N, Harrington W, Lasda E et al (2003) Of urchins and men: evolution of an alternative splicing unit in fibroblast growth factor receptor genes. RNA 9(2):209-217. doi:10.1261/rna. 2470903

24. Baraniak AP, Chen JR, Garcia-Blanco MA (2006) Fox-2 mediates epithelial cell-specific fibroblast growth factor receptor 2 exon choice. Mol Cell Biol 26(4):1209-1222. doi:10.1128/MCB. 26.4.1209-1222.2006

25. Thiery JP, Sleeman JP (2006) Complex networks orchestrate epithelial-mesenchymal transitions. Nat Rev Mol Cell Biol 7(2):131-142. doi:10.1038/nrm1835

26. Huber MA, Kraut N, Beug H (2005) Molecular requirements for epithelial-mesenchymal transition during tumor progression. Curr Opin Cell Biol 17(5):548-558. doi:10.1016/j.ceb.2005. 08.001

27. Lee JM, Dedhar S, Kalluri R et al (2006) The epithelial-mesenchymal transition: new insights in signaling, development, and disease. J Cell Biol 172(7):973-981. doi:10.1083/jcb.200601018

28. Chaffer CL, Thompson EW, Williams ED (2007) Mesenchymal to epithelial transition in development and disease. Cells Tissues Organs 185(1-3):7-19. doi:10.1159/000101298

29. Oltean S, Sorg BS, Albrecht T et al (2006) Alternative inclusion of fibroblast growth factor receptor 2 exon IIIc in Dunning 
prostate tumors reveals unexpected epithelial mesenchymal plasticity. Proc Natl Acad Sci USA 103(38):14116-14121. doi: 10.1073/pnas.0603090103

30. Hugo H, Ackland ML, Blick T et al (2007) Epithelial-mesenchymal and mesenchymal-epithelial transitions in carcinoma progression. J Cell Physiol 213(2):374-383. doi:10.1002/jcp. 21223

31. Gravdal K, Halvorsen OJ, Haukaas SA et al (2005) Expression of bFGF/FGFR-1 and vascular proliferation related to clinicopathologic features and tumor progress in localized prostate cancer. Virchows Archiv 448:1-7

32. Saha B, Arase A, Imam SS et al (2008) Overexpression of Ecadherin and beta-catenin proteins in metastatic prostate cancer cells in bone. Prostate 68(1):78-84. doi:10.1002/pros.20670

33. Subramanian A, Tamayo P, Mootha VK et al (2005) Gene set enrichment analysis: a knowledge-based approach for interpreting genome-wide expression profiles. Proc Natl Acad Sci USA 102(43):15545-15550. doi:10.1073/pnas.0506580102

34. Stanbrough M, Bubley GJ, Ross K et al (2006) Increased expression of genes converting adrenal androgens to testosterone in androgen-independent prostate cancer. Cancer Res 66(5):2815-2825. doi:10.1158/0008-5472.CAN-05-4000

35. Orengo JP, Bundman D, Cooper TA (2006) A bichromatic fluorescent reporter for cell-based screens of alternative splicing. Nucleic Acids Res 34(22):e148. doi:10.1093/nar/gk1967

36. Dunning WF (1963) Prostate cancer in the rat. Natl Cancer Inst Monogr 12:351-369

37. Lubaroff DM, Canfield L, Reynolds CW (1980) The Dunning tumors. Prog Clin Biol Res 37:243-263

38. Yan G, Fukabori Y, McBride G et al (1993) Exon switching and activation of stromal and embryonic fibroblast growth factor (FGF)-FGF receptor genes in prostate epithelial cells accompany stromal independence and malignancy. Mol Cell Biol 13(8): $4513-4522$

39. Landstrom M, Damber JE, Bergh A (1994) Prostatic tumor regrowth after initially successful castration therapy may be related to a decreased apoptotic cell death rate. Cancer Res 54(16):4281-4284

40. Feng S, Wang F, Matsubara A et al (1997) Fibroblast growth factor receptor 2 limits and receptor 1 accelerates tumorigenicity of prostate epithelial cells. Cancer Res 57(23):5369-5378
41. Matsubara A, Kan M, Feng S et al (1998) Inhibition of growth of malignant rat prostate tumor cells by restoration of fibroblast growth factor receptor 2. Cancer Res 58(7):1509-1514

42. Bonano VI, Oltean S, Brazas RM et al (2006) Imaging the alternative silencing of FGFR2 exon IIIb in vivo. RNA 12(12):2073-2079. doi:10.1261/rna.248506

43. Bonano VI, Oltean S, Garcia-Blanco MA (2007) A protocol for imaging alternative splicing regulation in vivo using fluorescence reporters in transgenic mice. Nat Protoc 2(9):2166-2181. doi: 10.1038/nprot.2007.292

44. Ramaekers FC, Verhagen AP, Isaacs JT et al (1989) Intermediate filament expression and the progression of prostatic cancer as studied in the Dunning R-3327 rat prostatic carcinoma system. Prostate 14(4):323-339. doi:10.1002/pros.2990140405

45. Mitic LL, Anderson JM (1998) Molecular architecture of tight junctions. Annu Rev Physiol 60:121-142. doi:10.1146/annurev. physiol.60.1.121

46. Chaffer CL, Dopheide B, McCulloch DR et al (2005) Upregulated MT1-MMP/TIMP-2 axis in the TSU-Pr1-B1/B2 model of metastatic progression in transitional cell carcinoma of the bladder. Clin Exp Metastasis 22(2):115-125. doi:10.1007/ s10585-005-5141-3

47. Wagner EJ, Baines A, Albrecht T et al (2004) Imaging alternative splicing in living cells. Methods Mol Biol 257:29-46

48. Seth P, Miller HB, Lasda EL et al (2008) Identification of an intronic splicing enhancer essential for the inclusion of FGFR2 exon IIIc. J Biol Chem 283:10058-10067

49. Thiery JP (2002) Epithelial-mesenchymal transitions in tumour progression. Nat Rev Cancer 2(6):442-454. doi:10.1038/nrc822

50. Thiery JP (2003) Epithelial-mesenchymal transitions in development and pathologies. Curr Opin Cell Biol 15(6):740-746. doi: 10.1016/j.ceb.2003.10.006

51. Tarin D, Thompson EW, Newgreen DF (2005) The fallacy of epithelial mesenchymal transition in neoplasia. Cancer Res 65(14):5996-6000 Discussion-1

52. Isaacs JT (1987) Development and characteristics of the available animal model systems for the study of prostatic cancer. In: Coffey DS, Bruchovsky N, Gardner WA, Resnick MI, Karr JP (eds) Current concepts and approaches to the study of the prostate. Alan R Liss, Inc., New York, pp 513-576 